\title{
NATURE OF BLUE ANTI-STOKES LUMINESCENCE IN ZnSe:Cr
}

\author{
V.Yu. IVANOV \\ Dnepropetrovsk State University, Dnepropetrovsk, Ukraine \\ Yu.G. Semenov \\ Institute of Semicond. Physics, Ukrainian Acad. Sciences, Kiev, Ukraine \\ M. Surma and M. Godlewski \\ Institute of Physics, Polish Academy of Sciences \\ Al. Lotników 32/46, 02-668 Warszawa, Poland
}

\begin{abstract}
The mechanism of the anti-Stokes photoluminescence in $\mathrm{ZnSe:Cr}$ is discussed. It is shown that the two-step ionization transitions of $\mathrm{Cr}\left(2^{+} \leftrightarrow 1^{+}\right)$ result in appearance of the blue anti-Stokes photoluminescence of $\mathrm{ZnSe:Cr}$. There are also some indications that the Auger type co-operative process can contribute to the photoluminescence excitation.
\end{abstract}

PACS numbers: $78.55 . \mathrm{Et}, 71.55 . \mathrm{Gs}, 76.30$.Fc

\section{Introductión}

Transition metal (TM) ions are efficient centers of nonradiative recombination in II-VI compounds [1]. In this paper we show that two-step ionization transitions of $\mathrm{Cr}$ result in the appearance of the donor-acceptor pair (DAP) photoluminescences (PLs) of $\mathrm{ZnSe}$ :Cr. Blue DAP PL of $\mathrm{ZnSe}$ is observed under photo-excitation with a photon energy lower than the emission energy. Such light emission is called anti-Stokes luminescence (ASL). Even though ASL was observed previously in several media [2-4] its mechanism is still disputed.

\section{Experimental results}

The PL spectrum of the $\mathrm{ZnSe:Cr}$ was measured upon different photo-excitation. For $2.807 \mathrm{eV}$ excitation (Fig. 1) sharp PL lines were observed; attributed to the radiative recombination of neutral donor bound exciton (DBE) and two acceptor bound excitons (ABE) and their LO phonon and two-hole (2s) replica. In the energy range between $2.6 \mathrm{eV}$ and $2.75 \mathrm{eV}$ PL spectrum consists of the two free-to-bound (electron-shallow acceptors) and of the shallow donor ( $\mathrm{Ga}$ or $\mathrm{Al}$ ) 


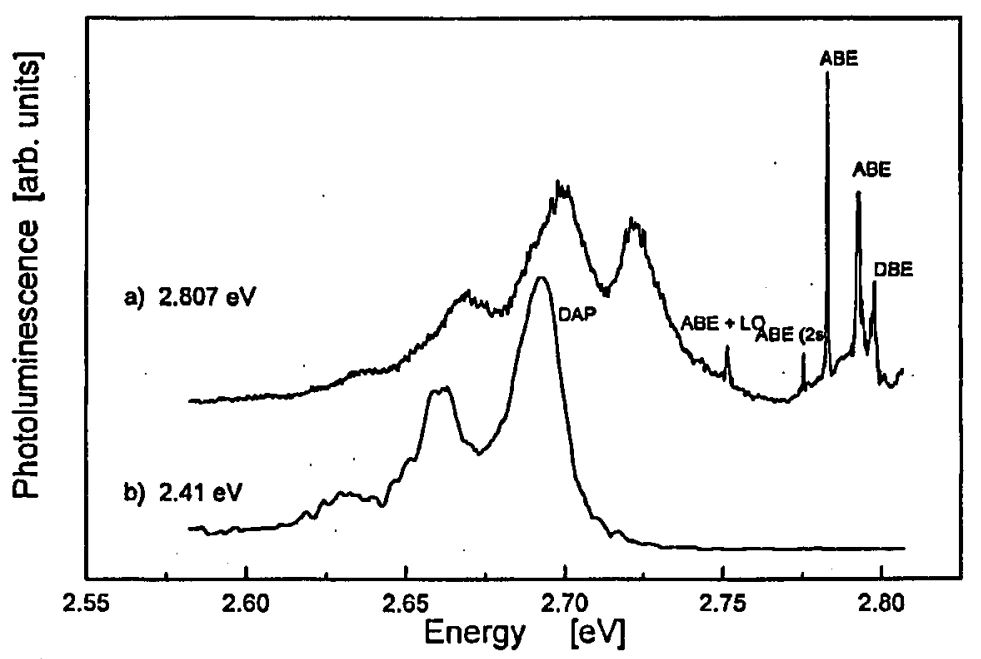

Fig. 1. "Edge" part of the low temperature photoluminescence spectrum of ZnSe measured under band to band (a) and $2.41 \mathrm{eV}$ photo-excitation (b).

to shallow acceptor $(\mathrm{Li})$ transitions. This part of spectrum depends strongly on excitation intensity (PL shifts towards higher energy) and on the temperature (above $60 \mathrm{~K}$ DAP PL is deactivated and free-to-bound PL dominates). The blue DAP emission is observed regardless excitation energy. It is also observed for the excitation energy smaller than the emission energy. The light intensity dependence of the ASL is roughly linear. Small intensity hysteresis was, however, observed. We have also observed well known red and green DAP PLs of ZnSe. Their intensity strongly depended on the photo-excitation energy.

Electron spin resonance (ESR) spectrum obtained under photo-excitation (photo-ESR) consisted of weak $\mathrm{Fe}^{3+}$ and strong $\mathrm{Cr}^{+} \mathrm{ESR}$ signals. The photo-excitation spectrum of the Cr${ }^{+}$ESR signal was measured and is compared in Fig. 2 with the photo-excitation of the blue DAP ASL emission of $\mathrm{ZnSe}: \mathrm{Cr}$.

\section{Discussion}

Two mechanisms were proposed to explain ASL in semiconductors. The first is a multi-photon (two-photon) transition and the second is a two-step ionization transition via some deep center. Our results favor the latter mechanism of the ASL in ZnSe:Cr. The ASL is excited (see Fig. 2) for photon energies larger than $2 \mathrm{eV}$, i.e., for $\mathrm{Cr}^{2+} \rightarrow \mathrm{Cr}^{+}$ionization transitions identified previously [5]. Once $\mathrm{Cr}^{+}$is populated, the $\mathrm{Cr}^{2+} \rightarrow \mathrm{Cr}^{2+}$ transition is also possible. Free holes are created in the valence band $(\mathrm{VB})\left(2^{+} \rightarrow 1^{+}\right.$transition $)$and free electrons are photo-generated in the conduction band (CB) $\left(1^{+} \rightarrow 2^{+}\right.$transition) and some of them are trapped by shallow donors and by acceptors active in the DAP PL transitions [6]. As a result the ASL DAP PL can be observed.

Both two-photon and two-step ionization transitions should lead to a $I^{2}$ dependence of the ASL intensity $\left(I_{\mathrm{PL}}\right)$ on the excitation intensity $(I)$. However, 


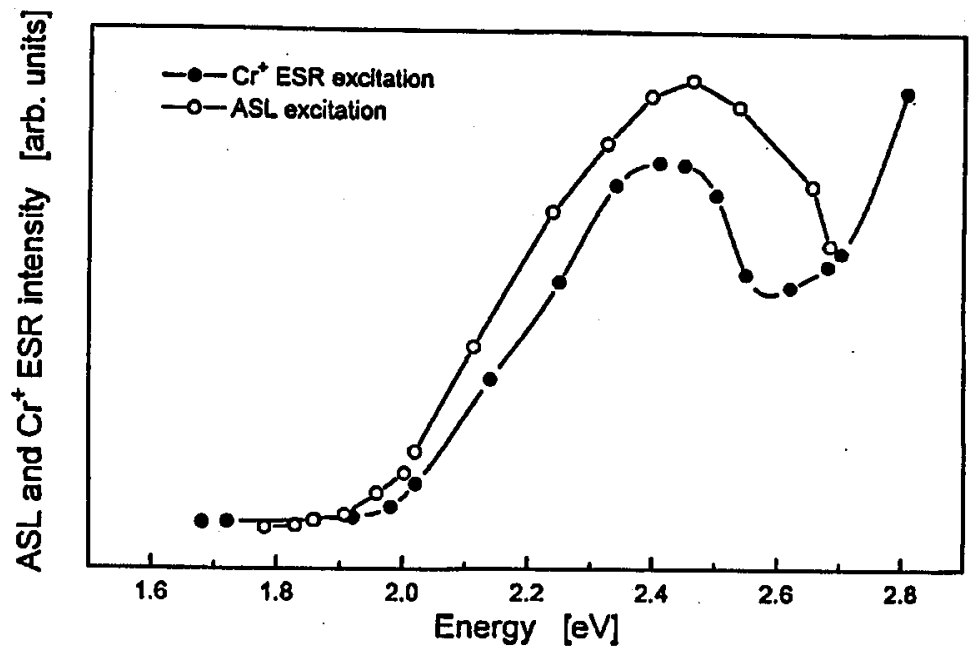

Fig. 2. Excitation spectra of the blue DAP PL (•) and of the ESR signal of the Cr $\mathbf{C r}^{+}$ (). The ESR excitation spectrum consists of the two bands attributed previously [5, 9] to the direct ionization transition of $\mathrm{Cr}$ and (higher energy band) to the indirect process in which acceptor centers are first ionized.

a linear dependence was observed in our case. To explain such dependence of the $I_{\mathrm{PL}}$ we have solved a set of the kinetics equations describing recombination transitions in $\mathrm{ZnSe}: \mathrm{Cr}$ under $\mathrm{Cr}$ ionization excitation. The $I_{\mathrm{PL}} \approx$ const $I N_{\mathrm{Cr}}\left(N_{\mathrm{Cr}}\right.$ is $\mathrm{Cr}$ concentration) dependence of the DAP ASL intensity is explained assuming efficient trapping of free holes by shallow acceptors or/and $\mathrm{Cr}$-acceptor tunneling transitions.

In the $\mathrm{Cr}$ ionization processes both free electrons and holes are created as in the band-to-band photo-excitation. However, Fig. 1 shows distinct differences in the PL spectrum observed in these two cases. Bound excitonic PLs are absent in the ASL. For the VB $\rightarrow$ CB excitation free electrons and holes appear "simultaneously". They can form free excitons or be trapped at donors and/or acceptors resulting in the DBE and ABE PLs. For the two-step excitation free carriers are photo-generated "separately". Moreover, deep trap levels due to TM ions are charged increasing their role in free carriers trapping [1]. Figure 1 indicates that excitons are not formed under such excitation. Free-to-bound PL was also missing in the ASL.

The relative intensity of the red and green DAP PLs [7] depended on the photo-excitation energy. The green PL appeared only under the direct photo-ionization of the $0.4 \mathrm{eV}$ acceptor, whereas the red PL was also observed under $\mathrm{Cr}$ photo-ionization. A new type of the co-operative transition can result in the photo-excitation of this PL band. We propose that the Auger type energy transfer process can be responsible for the excitation of the red $\mathrm{PL}$ in $\mathrm{ZnSe} \mathrm{Cr}$. Three center Auger transitions were observed in previous ESR studies of $\mathrm{ZnS}$ and $\mathrm{ZnSe}$ $[8,9]$. In the process the DAP recombination energy is transferred to a nearby TM 
and used to ionize the ion. For Fe in $\mathrm{ZnS}$ "hot" electrons were then generated in the $\mathrm{CB}$, which thermalized rapidly making the reverse process $\left(\mathrm{Fe}^{3+}\right.$ traps an electron and energy is used for the DAP excitation) inefficient. However, for $\mathrm{Cr}$ ion in $\mathrm{ZnSe}$ the reverse transition should be possible since the energy of the $\mathrm{Cr}^{2+} \rightarrow \mathrm{Cr}^{+}$ ionization transition is nearly equal to the DAP excitation energy of the red PL. Even though we have no direct proof for a high efficiency of the above transition, we believe that such co-operative processes are important in $\mathrm{ZnSe}: \mathrm{Cr}$ and lead to a resonant excitation of the red DAP PL.

This work was partly supported by grant $0549 / \mathrm{P} 3 / 94 / 07$ of the State Committee for Scientific Research (Republic of Poland).

\section{References}

[1] M. Godlewski, A. Zakrzewski, in: II-VI Semiconductors, Ed. M. Jain, World Scientific, Singapore 1993, p. 205.

[2] D.L. Dexter, Phys. Rev. 126, 1962 (1962).

[3] P.P. Feofilov, V.V. Ovsyankin, Appl. Opt. 6, 1828 (1967).

[4] I. Broser, R. Broser-Warminsky, in: Proc. Int. Conf. Luminescence of Organic and Inorganic Materials, New York 1962, p. 402.

[5] M. Godlewski, M. Kamińska, J. Phys. C 13, 6537 (1980).

[6] M. Godlewski, Phys. Status Solidi A 90, 11 (1985).

[7] M. Godlewski, W.E. Lamb, B.C. Cavenett, J. Lumin. 24/25, 173 (1981).

[8] A. Zakrzewski, M. Godlewski, Phys. Rev. B 34, 8993 (1986).

[9] M. Surma, A. Zakrzewski, M. Godlewski, T.P. Surkova, Acta Phys. Pol. A 87, 221 (1995). 